\title{
Why We Should Be Skeptical of Quantum Computing
}

This paper was downloaded from TechRxiv (https://www.techrxiv.org).

\section{LICENSE}

CC BY 4.0

SUBMISSION DATE / POSTED DATE

$12-11-2021 / 17-11-2021$

CITATION

Kadin, Alan (2021): Why We Should Be Skeptical of Quantum Computing. TechRxiv. Preprint. https://doi.org/10.36227/techrxiv.17003635.v1

$\mathrm{DOI}$

10.36227/techrxiv.17003635.v1 


\title{
Why We Should Be Skeptical of Quantum Computing
}

\author{
Alan M. Kadin \\ Consultant \\ Princeton Junction, NJ USA \\ amkadin@alumni.princeton.edu
}

\begin{abstract}
It is widely believed that quantum computing is on the threshold of practicality, with performance that will soon greatly surpass that of classical computing. On the contrary, I argue that quantum computing does not currently exist, and probably never will. First, although quantum annealing systems have been demonstrated to solve practical optimization problems, they are actually performing classical analog annealing, with no quantum enhancement. In contrast, while systems of quantum gate arrays, which are expected to perform digital quantum computing, have been fabricated with up to $\sim 100$ qubits in several technologies, they have not performed any practical computations. This is not merely a question of excess noise; the theory of massive quantum entanglement, necessary for the desired performance, has never been actually been verified. The well-established quantum results such as electronic energy bands do not incorporate quantum entanglement. I suggest that the experimental observations in multi-qubit systems may be explained as the result of delocalized coupled oscillator modes, similar to that in electronic energy bands. Such coupled modes would not yield the exponential increase in degrees of freedom needed for quantum speedup, and hence would not be useful for computing. Tests on these multi-qubit systems should be able to distinguish these two models. The quantum computing research community really needs to address this issue.
\end{abstract}

Keywords-quantum computing, simulated annealing, analog computers, parallel processing, quantum entanglement, cryogenic electronics.

\section{THE EXTRAORDINARY CLAIMS OF QUANTUM COMPUTING}

Carl Sagan once said [1], "Extraordinary claims require extraordinary evidence." The claims of quantum computing for future exponential parallelism in computer performance are indeed extraordinary, and these claims have been largely accepted by most of the computing community, with little evidence. Governments, corporations, and investors around the world are pouring billions of dollars into this field. This is a hype cycle [2], where the hype has been greatly inflated by identifying quantum computing as a critical technology for national security. I predict that the entire field will collapse, probably within five years, with little to show for the efforts.

In any case, researchers in the new field of quantum computing should investigate the scientific basis for these predictions prior to large-scale engineering development, but this has never been done. Indeed, serious research papers $[3,4]$ that have expressed skepticism and proposed such investigations have been blocked from publication in journals and conference proceedings. One exception is an article in IEEE Spectrum [5], questioning whether quantum computing can ever be successful.

The primary reason for the predicted power of quantum computing is the phenomenon of quantum entanglement [6]. For a set of $\mathrm{N}$ classical bits, there are $2^{\mathrm{N}}$ distinct configurations, but only one at any given time. In contrast, according to the theory of quantum computing, if $\mathrm{N}$ quantum bits (qubits) are fully entangled, all $2^{\mathrm{N}}$ states may be present at the same time (as superpositions). An operation that acts on this entangled state effectively operates on all $2^{\mathrm{N}}$ states in parallel. This parallelism grows exponentially, much faster any conceivable hardware parallelism with classical bits. For example, when $\mathrm{N}=300,2^{\mathrm{N}}$ is larger than the number of atoms in the known universe.

Computationally difficult algorithms are central to modern cryptography. For example, the standard RSA public key encryption system depends on the practical inability to factor large integers. However, Shor's algorithm [7] (1994) showed how a quantum computer could in principle factor very large integers, and that was when the US National Security Agency started to support research into quantum computing.

There are at least two problems with this effort, however. First, these highly entangled states are exquisitely sensitive to low levels of noise, which introduce quantum errors. It is widely believed that extensive quantum error correction will be required, and will substantially dominate the circuits. Cooling to deep cryogenic temperatures is not enough. Other experts $[5,8]$ have argued that this quantum error correction will be impossible at the required 
scale, making the entire enterprise problematic. Some researchers have identified possible applications for Noisy Intermediate Scale Quantum technology (NISQ) [9], but this remains an open question.

The second fundamental problem is that the required massive entanglement has never been demonstrated, and is not responsible for those aspects of quantum theory that are well established. For example, Schrödinger's equation and electronic band theory do not depend on entanglement. Indeed, Schrödinger and Einstein were early critics of entanglement in the 1930s [6]. Much later in the 1970s, entanglement was demonstrated in pairs of photons, but massive entanglement was never tested. I argue that tests of the entanglement model of quantum computing are long overdue, and some are suggested in this article.

\section{CLASSICAL AND QUANTUM ANNEALING}

It is important to understand that there are a variety of architectures and technologies that advertise themselves as "quantum computing", and some are quite different from the others. Most are designed around digital quantum gates, where the gates may be constructed in a range of different technologies: superconducting Josephson junctions, ions, photons, quantum dots. All of these promise exponential parallelism based on entanglement, and none of these is designed to function as a classical (non-quantum) gate. Thus far, none of these have demonstrated performance of a significant computational problem.

In contrast, a "Quantum Annealer" is already a commercial product (from D-Wave Systems, Inc.), which has been demonstrated to solve combinatorial optimization problems [10]. This is based on superconducting Josephson junctions, and may therefore appear to be similar to the superconducting digital quantum gate systems being developed by other groups (such as IBM and Google). However, the quantum annealer is really an analog computer designed to emulate the classical computational method known as simulated annealing [11], based on the Ising model. Simulated annealing can be used to solve a wide range of optimization problems, of which the traveling salesman problem is the benchmark. Furthermore, the circuits are configured to act as an analog annealer even in the fully classical limit, in the absence of any quantum effects. So the successful operation of this quantum annealer does not validate the generic quantum computing approach.

It has been asserted that a quantum annealing system offers improved performance over a purely classical annealing system, via a process known as quantum tunneling. However, this benefit has not been clearly validated [12], but quantum annealing does not seem to offer the exponential parallelism that motivates entanglement-based quantum computing.

Classical simulated annealing may be carried out on a standard classical von Neumann processor, but that may be quite slow for optimization with large numbers of variables. There have recently been a number of custom classical Ising processors in a wide range of alternative architectures for improved performance. One is available commercially from Fujitsu, called a "digital annealer" [13]. This is a standard CMOS digital chip operating at room temperature, rather than any novel technology. Fujitsu markets this as "quantum-inspired computing technology", but there is nothing quantum about this. The superior performance is associated with the integrated memory, parallelism, and interconnect density in a non-von-Neumann architecture. Similarly, Hitachi has a "CMOS annealer" [14], and several research groups have demonstrated "coherent Ising processors" based on integrated optics [15].

All of these annealing machines are addressing the same market for optimization algorithms, and they could compare performance on standard benchmarks such as the traveling salesman problem [16]. Unless quantum annealing can establish clearly superior performance, it is difficult to see how a cryogenic system requiring cooling to a temperature of $20 \mathrm{mK}$ can compete with scalable CMOS chips at room temperature.

\section{CLASSICAL AND QUANTUM BITS}

Classical digital computers are based on arrays of bits, and classical digital circuits are based on arrays of bit slices. Each bit is a bistable device with transitions between ' 0 ' and ' 1 ' that require a signal much larger than the noise level. This is typically a heavily damped system that permits a rapid transition with no ringing. Multiple bits do not interact with one another; operations on a given bit slice will not affect others, apart from carry bits. An N-bit system will have $2^{\mathrm{N}}$ possible states, but each state is definite and all transitions are deterministic. These rules apply not only to conventional semi-conductor transistors, but also to alternative technologies such as Josephson junctions [17]. Josephson junctions are naturally high-Q LC resonators, but damping resistors are deliberately added to RSFQ classical digital logic circuits to suppress oscillations and increase switching speed. This also reduces uncontrolled coupling between adjacent bits.

In contrast, a qubit consists of a high- $Q$ resonator that can be in either of two states, labeled $|0\rangle$ and $|1\rangle$, with a transition in either direction induced by a photon at the resonant frequency, typically in the microwave range. But according to the qubit theory, these two are not the only options. More generally, a qubit can be in a linear 
superposition of $|0\rangle$ and $|1\rangle$, representing the relative probability of measuring the qubit in one state or the other. It is widely believed that Josephson junctions exhibit macroscopic quantum effects at ultralow temperatures [18], although other researchers have indicated that most of this behavior is really classical $[19,20]$.

Furthermore, qubits are designed to interact weakly with one another, and the multi-qubit state is in a linear combination of all $2^{\mathrm{N}}$ qubit configurations. So an operation on any qubit will affect all other coupled qubits. This is a manifestation of quantum entanglement, and is different from classical computing. Further, there can be no noise or perturbation during the time these operations occur; these entangled states are very sensitive, and are subject to "decoherence".

The term "quantum supremacy" has been used to imply that state-of-the-art quantum computers are already more powerful than classical computers [21,22]. This is highly misleading, in that these quantum computers have not been shown to perform any real computation faster than a classical computer. Instead, this term has been used to describe the simulation of the fully entangled theoretical states on a classical computer. If the number of entangled qubits is larger than about 50 , the effective parallelism of $2^{\mathrm{N}}$ is so large that a classical computer cannot calculate it in a reasonable time. That does not, of course, prove that the quantum computer is operating as an ideal entangled state, particularly given the presence of noise.

It should be pointed out that this quantum entanglement theory is not the same quantum theory that has been used for decades to compute energy bands in semiconductor crystals [23]. Bloch wave theory is well known to produce delocalized effects without any entanglement, and is actually due to collective modes of classical oscillators. Remarkably, quantum computing researchers never cite the established quantum theory that may explain their measurements. Coupled mode theory is described further in the next section.

\section{CLASSICAL AND QUANTUM COUPLED MODES}

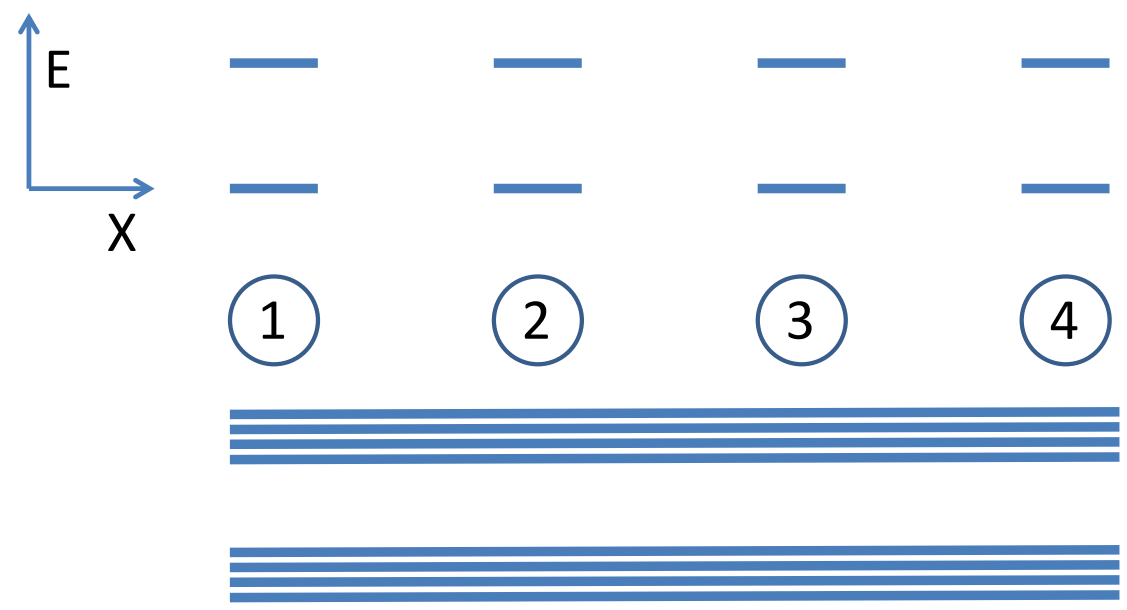

Fig. 1. Delocalized coupled modes in arrays of atoms or qubits.

Consider Fig. 1, with 4 identical atoms being brought together so that they interact in a linear crystal. Each atom has a localized ground electron state and an excited state. It is an established consequence of quantum mechanics that each localized state gives rise to an energy band of delocalized states. This does not increase the number of degrees of freedom; 4 atoms give rise to 4 delocalized states in the each energy band. Weak nearest-neighbor interactions give rise to narrow bands; stronger interactions cause broader bands. The extended electron wave functions are known as Bloch waves [24], may be approximated as linear combinations of atomic orbitals, and calculated from the periodic potential of the atoms. The atomic orbitals no longer exist in the crystal; they are replaced by the Bloch waves.

More generally, for a large number $\mathrm{N}$ atoms in a crystal, the $\mathrm{N}$ states in each energy band become much closer together, but in principle they remain discrete. The initial $2 \mathrm{~N}$ localized states become $2 \mathrm{~N}$ Bloch waves. There is no entanglement in this theory, which gives rise to energy bands in semiconductor crystals. There can also be localized electronic states in a crystal, associated with localized impurity atoms that have a frequency mismatch with the surrounding states. 
This theory of Bloch waves represents an application to coupled electrons of a more general classical theory of coupled modes. Consider, for example, coupled mechanical oscillators (a network of springs), or coupled electromagnetic oscillators. A localized excitation will not stay localized; it will quickly propagate through the system. Collective modes are well known in classical periodic systems, and form frequency bands.

All qubit systems represent coupled quantum resonators. They may be electronic states (as with ions or quantum dots), or electromagnetic resonators (as with Josephson junctions), but if coupled together, they will form Bloch waves and energy bands. Could Bloch wave theory account for measured behavior in arrays of qubits, rather than the entanglement theory? Some preliminary evidence suggests that this may be the case, but more quantitative comparison is necessary. If true, this would have major implications for the future of quantum computing.

\section{TESTING THE QUANTUM ENTANGLEMENT MODEL}

Consider a linear array of coupled qubits with tunable energy splittings and couplings. If the array is uniform, this would correspond to a perfect crystal, and would permit Bloch wave propagation across the entire array. If, however, the qubit in the center is detuned, this would correspond to an impurity in the crystal that would block propagation of the Bloch wave.

An experiment of this type was reported based on an array of 9 superconducting transmon qubits, each coupled to a common transmission line [21]. For a uniform qubit array, a resonant excitation at one end was detected at the other end. For an array with a detuned qubit at its center, a resonant excitation at one end was not detected at the other end. This was successfully modeled with the entanglement model. But is this the unique explanation? If it turns out that the Bloch wave model can quantitatively account equally well for the measurements, then this experiment cannot be used to provide evidence for either model.

The Bloch wave model should be far easier to simulate than the entanglement model, particularly for large arrays where the entanglement model requires an exponential expansion in the effective dimensionality. The Bloch wave model should be included in every quantum computing analysis, and researchers should identify measurements where they make distinctly different predictions. In particular, there should be a way for the expected exponential expansion with the number of qubits to be monitored.

In summary, the predictions of the entanglement model of quantum computing are truly extraordinary, promising exponentially large effective parallelism. What is needed is equally extraordinary evidence, which rules out other explanations such as the Bloch wave model. This has not yet been explored, but could and should be done before full engineering scaleup. In the meantime, we should all be skeptical of the promises of quantum computing.

\section{REFERENCES}

[1] Wikipedia, "Sagan Standard", retrieved August 2021, https://en.wikipedia.org/wiki/Sagan_standard.

[2] Wikipedia, "Hype Cycle", retrieved August 2021, $\quad$ https://en.wikipedia.org/wiki/Hype cycle.

[3] A.M. Kadin and S.B. Kanlan. "Sunerconducting Ouantum Comnuting Without Entanglement?", Submitted to Applied Superconductivity Conference, 2014, Unpublished. Available online at https://vixra.org/abs/1409.0001.

[4] A.M. Kadin and S.B. Kanlan. "Proposed Experiments to Test the Foundations of Ouantum Computing". Suhmitted to International Conference on Rebooting Computing, 2016, Unpublished. Available online at https://vixra.org/abs/1607.0105.

[5] M. Dyakonov, "The Case Against Quantum Computing”, IEEE Spectrum, November 2018, https://spectrum.ieee.org/the-case-against-quantum-computing.

[6] Wikinedia. "Ouantum Entanglement". retrieved August 2021, https://en.wikipedia.org/wiki/Quantum_entanglement.

[7] Wikipedia, "Shor's Algorithm", retrieved August 2021, https://en.wikipedia.org/wiki/Shor's_algorithm.

[8] G. Kilai, "The Quantum Computer Puzzle", Unpublished, 2016. Available online at https://arxiv.org/abs/1605.00992.

[9] J. Preskill, "Quantum computing in the NISQ era and beyond", Quantum Journal, 2018. Available online at https://quantum-journal.org/papers/q-2018-08-06-79/ .

[10] D-Wave Systems, "What is Quantum Annealing", retrieved August 2021 at https://docs.dwavesys.com/docs/latest/c_gs_2.html.

[11] Wikinedia. "Simulated Annealing". retrieved August 2021, https://en.wikipedia.org/wiki/Simulated_annealing.

[12] J. Smolin and G. Smith, "Classical signature of quantum annealing," Frontiers in Physics (2014). Available online at https://www.frontiersin.org/articles/10.3389/fphy.2014.00052/full

[13] J. Boyd, "Silicon Chip Delivers Quantum Speeds", IEEE Spectrum, July 2018. Available online at https://spectrum.ieee.org/fujitsus-cmos-digital-annealer-produces-quantum-computer-speeds. 
[14] Y. Masanao, “CMOS Annealing Machine,” Hitachi Research \& Development, November $2018 . \quad$ Available online at https://www.hitachi.com/rd/sc/story/cmos_annealing2/index.html.

[15] J. Hsu, "New Computer Combines Electronic Circuits with Light Pulses," IEEE Spectrum, October 2016. Available online at https://spectrum.ieee.org/new-computer-combines-electronic-circuits-with-light-pulses.

[16] R. Hamerly, et al., "Experimental investigation of performance differences between coherent Ising machines and a quantum annealer," Science Advances, vol. 5, May 2019. Available online at https://advances.sciencemag.org/content/5/5/eaau0823

[17] D.S. Holmes, et al., "Superconducting Computing in Large-Scale Hybrid Systems," IEEE Computer, vol. 48, p. 34, December 2015. https://ieeexplore.ieee.org/abstract/document/7368011

[18] M.H. Devoret, et al., "Measurements of macroscopic quantum tunneling out of the zero-voltage state of a current biased Josephson junction," Phys. Rev. Lett., vol. 55, p. 1908, 1985. Available online at https://journals.aps.org/prl/abstract/10.1103/PhysRevLett.55.1908

[19] J.A. Blackburn, et al., "A survey of classical and quantum interpretations of experiments on Josephson junctions at very low temperatures," Physics Reports, vol. 611, p.1, 2016. Available online at https://arxiv.org/abs/1602.05316.

[20] N. Gronbech-Jensen, et al., "Tomography and entanglement in coupled Josephson junction qubits," Phys. Rev. Lett., vol. 105, 010501, 2010. Available online at https://journals.aps.org/prl/abstract/10.1103/PhysRevLett.105.010501

[21] C. Neill, et al., "A blueprint for demonstrating quantum supremacy with superconducting qubits," Science, vol. 360, p. 195, 2018. https://science.sciencemag.org/content/360/6385/195

[22] F. Arute, et al., "Quantum Supremacy using a Programmable Superconducting Processor," Nature, vol. 574, p. 505, 2019. https://www.nature.com/articles/s41586\%20019\%201666\%205

[23] Wikinedia. "Flectronic Band Structure", retrieved August 2021, https://en.wikipedia.org/wiki/Electronic_band_structure.

[24] Wikipedia, “Bloch's Theorem”, retrieved August 2021, https://en.wikipedia.org/wiki/Bloch's_theorem. 